\title{
MANEJO DE LA ENFERMEDAD HEPÁTICA METABÓLICA GRASA
}

\section{MANAGEMENT OF FAT METABOLIC HEPATIC DISEASE}

\author{
Lucena A', Sánchez Torrijos Y', Ampuero Herrojo J²
}

'Servicio de Aparato Digestivo. Hospital Universitario Virgen del Rocío. Sevilla.

${ }^{2}$ Servicio de Aparato Digestivo. Hospital Universitario Virgen del Rocío. Sevilla. Instituto de Biomedicina de Sevilla. Centro de Investigación Biomédica en Red de Enfermedades Hepáticas y Digestivas. Universidad de Sevilla.

\section{Resumen}

La enfermedad hepática metabólica grasa es la primera causa de hepatopatía crónica a nivel mundial, y se prevé que el número de casos aumente considerablemente en los próximos años, debido al estilo de vida actual. Su prevalencia se encuentra positivamente correlacionada con el síndrome metabólico, así como con cada una de sus variables. Asimismo, también es destacable su asociación con numerosas enfermedades inmunomediadas.

Aunque el diagnóstico definitivo continúa siendo histológico, se han desarrollado numerosos índices serológicos que determinan el riesgo de presentar tanto esteatosis hepática como fibrosis, principal factor pronóstico de la evolución de la enfermedad. Estos métodos no invasivos son apoyados por diferentes técnicas de imagen, entre las que destaca la elastografía transitoria.

La principal estrategia terapéutica en la esteatosis hepática metabólica es la intervención en el estilo de vida. Las intervenciones más comunes están centradas en la pérdida de peso, siendo la dieta mediterránea la más beneficiosa y eficaz, así como en la realización de una actividad física moderada de forma habitual. No existe un tratamiento farmacológico aprobado en la actualidad. Sin embargo, existen numerosos tratamientos en régimen de ensayos clínicos en fase avanzada, entre los que destacan ácido obeticólico, elafibranor,

\section{CORRESPONDENCIA}

Javier Ampuero Herrojo

Hospital Universitario Virgen del Rocío

41013 Sevilla

jampuero-ibis@us.es

Fecha de envío: 18/02/2020

Fecha de aceptación: 01/03/2020 y liraglutide. En este artículo revisamos los métodos diagnósticos más utilizados, así como el manejo posterior que se debe llevar a cabo en estos pacientes.

Palabras clave: enfermedad hepática metabólica grasa, esteatosis hepática, esteatohepatitis, fibrosis hepática.

\section{Abstract}

Non-alcoholic fatty liver disease (NAFLD) is the leading cause of chronic liver disease in Western countries, with a growing incidence in the coming years. Its prevalence is positively correlated to the metabolic syndrome and, consequently, to the single components. Besides, a strong association with numerous immunomodulated diseases has also been reported.

Although the definitive diagnosis of NAFLD is based on histology, different non-invasive serum tests have been developed to determine the presence of liver steatosis and fibrosis, which represents the main prognostic factor of this entity. Besides, there are non-invasive imaging methods, based predominantly on elastography, which can complete the diagnosis and grading the disease.

Lifestyle intervention is the main therapeutic strategy in NAFLD. It is focused on weight loss, changing the diet pattern (Mediterranean diet is the most beneficial and useful), and performing a frequent moderate physical activity. Currently, there is no pharmacological treatment approved for NAFLD. However, plenty of drugs are currently being tested in randomized clinical trials. In this article, we review an overall approach on NAFLD, ranging from the most used diagnostic methods to the subsequent therapeutic management that should be carried out in these patients. 
Keywords: non-alcoholic fatty liver disease steatosis, steatohepatitis, liver fibrosis.

\section{Introducción}

La enfermedad hepática metabólica grasa (EHMG) es la patología hepática más prevalente a nivel mundial, con un $25 \%$ de población afecta ${ }^{1}$. Engloba un amplio espectro de lesiones hepáticas, que abarcan desde la esteatosis simple a la esteatohepatitis. Mientras que la esteatosis simple es una entidad generalmente benigna, la esteatohepatitis puede progresar a fibrosis ocasionando secundariamente cirrosis con sus consecuentes complicaciones. Exceptuando una leve alteración de las enzimas de función hepática, carece de síntomas hasta estadios avanzados. En este punto radica la trascendencia de la sospecha clínica en pacientes que presenten factores riesgo asociados a EHMG.

\section{Cuándo sospechar EHMG}

Se encuentra ampliamente descrita la estrecha relación con el síndrome metabólico (SMet). De hecho, la prevalencia de EHMG aumenta paralelamente con la del SMet, tanto en su conjunto como con cada una de sus variables de forma independiente, en especial la obesidad ${ }^{2}$ y diabetes mellitus (DM). Hasta en el $50-70 \%$ de los pacientes con obesidad se ha demostrado la existencia de EHMG, siendo el porcentaje mayor en aquellos con obesidad de predominio abdominal. La acumulación de grasa visceral y la disfuncionalidad del tejido adiposo en sujetos con un índice de masa corporal dentro de la normalidad se ha correlacionado con una mayor incidencia de $\mathrm{EHMG}^{3}$. Además, hasta el $95 \%$ de los pacientes sometidos a cirugía bariátrica presentan esta entidad ${ }^{4}$. En el caso de (DM), la prevalencia oscila entre el $60-80 \%{ }^{5,6}$, relacionándose a su vez con la gravedad de la enfermedad hepática. Dada la asociación bidireccional existente entre ambas entidades, es preceptivo descartar DM en los pacientes con EHMG mediante la evaluación anual de Hb1Ac. En aquellos casos donde coexisten DM y obesidad, la prevalencia de NAFLD es casi universal ${ }^{3}$. Por otro lado, hasta un $50 \%$ de los pacientes con dislipemia presentan también $\mathrm{EHMG}^{7}$.

Si bien los componentes del síndrome metabólicos son los factores de riesgo fundamentales para la esteatosis hepática, también está descrita en pacientes con normo e incluso bajo peso. En este contexto, es importante resaltar la relación entre EHMG y numerosas enfermedades inmunomediadas. Un ejemplo de esto, es la enfermedad inflamatoria intestinal (EII). Se ha demostrado un aumento de incidencia de EHMG en pacientes con EII, principalmente en los casos con evolución más larga de enfermedad intestinal, independientemente de la edad al diagnóstico ${ }^{8}$. En los pacientes con Ell, la EHMG presenta menor asociación con factores de riesgo metabólico sugiriendo así que el desarrollo de la patología hepática pueda estar influenciada por la actividad inflamatoria de la propia Ell más que con los factores metabólicos tradicionalmente descritos ${ }^{9,10}$. Otros factores de riesgo independientes en la Ell son la edad avanzada, los factores de riesgo metabólico, el uso de metotrexato y la cirugía previa ${ }^{11}$. En algunas publicaciones se ha propuesto que una Ell de curso grave se relaciona con mayor riesgo de fibrosis hepática ${ }^{12}$.

Otra relación con enfermedades inmunomediadas descrita es la enfermedad psoriásica, donde se ha objetivado una incidencia
1,5-3 veces mayor de EHGM que en el resto de la población ${ }^{13}$. Además, una enfermedad dermatológica de características agresivas se ha correlacionado con un mayor riesgo de fibrosis, similar a lo que ocurre con la $\mathrm{EII}^{14}$. El vínculo patogénico entre la psoriasis y la EHMG es la inflamación crónica y la resistencia periférica a la insulina. Este es el punto común fundamental de relación con otras numerosas enfermedades que están siendo actualmente descritas, como el síndrome de ovario poliquístico ${ }^{15}$ o la hidradenitis supurativa ${ }^{16}$.

\section{Cómo evaluar de forma no invasiva la EHMG}

El gold estándar para el diagnóstico definitivo de EHMG continúa siendo la biopsia hepática. Además, se trata de la única técnica por el momento capaz de detectar la presencia de esteatohepatitis, usando el NAS score (que representa la suma de los grados de esteatosis, balonización e inflamación lobulillar). No obstante, se trata de una técnica invasiva, no exenta de riesgos, que presenta como limitación fundamental la variabilidad interobservador ${ }^{17,18}$. Por este motivo, se debe realizar únicamente en pacientes seleccionados para confirmación diagnóstica, descartando otras posibles causas de hepatopatía ${ }^{19}$. En este escenario, surge la necesidad de desarrollar alternativas diagnósticas no invasivas, basadas en biomarcadores y pruebas de imagen, con el objetivo de identificar el riesgo de EHMG y su gravedad (reduciendo la necesidad de realizar estudio histológico).

\section{Esteatosis hepática}

No existen determinaciones analíticas aisladas que permitan detectar esteatosis hepática. La elevación de transaminasas, marcador comúnmente usado como dato de sospecha de hepatopatía, puede estar elevado hasta 2-3 veces respecto a su valor normal. Sin embargo, la normalidad de las mismas no excluye la existencia de EHMG. De hecho, existe una normalidad de la bioquímica hepática en un gran número de pacientes con fibrosis avanzada y cirrosis hepática ${ }^{20,21}$, en probable relación a la disminución gradual de las reacciones necroinflamatorias en las fases avanzadas de fibrosis.

La cuantificación de la esteatosis hepática carece de utilidad en la práctica clínica habitual, a excepción de la valoración de respuesta al tratamiento en ensayos clínicos. A pesar de ello, se han desarrollado numerosos índices séricos difícilmente comparables entre sí por haberse validado con estándares diferentes (biopsia hepática, ecografía o RM) ${ }^{22}$. De ellos, el Fatty Liver Index y el Hepatic Steatosis Index se han validado de forma independiente con valores de AUROC similares para el diagnóstico de esteatosis ${ }^{23}$. Un inconveniente de estos tests es que predicen la existencia o ausencia de esteatosis pero no la cuantifican.

La ecografía es una técnica de imagen inocua utilizada para la detección de esteatosis hepática. Se observa un aumento de la ecogenicidad del parénquima hepático en relación con la corteza renal. Sin embargo, la sensibilidad diagnóstica de la misma se reduce notablemente cuando la infiltración grasa es menor del $30 \%{ }^{24,25}$, así como en los pacientes que presentan obesidad ${ }^{26}$. Por tanto, la ausencia de la misma no descarta la existencia de EHMG en presencia de factores de riesgo bien conocidos. 
Otra herramienta capaz de confirmar o descartar la esteatosis es el Controlled Attenuation Parameter (CAP). EI CAP se basa en la atenuación del ultrasonido a través del hígado en una medición que se realiza de forma simultánea a la elastografía transitoria, de forma que se correlaciona con la infiltración de grasa hepática. Recientemente se han propuesto límites $248 \mathrm{~dB}$ para identificar una esteatosis $>5 \% 27$.

Finalmente, existen varias técnicas mediante resonancia magnética (RM) para la cuantificación de grasa hepática. La espectroscopia por resonancia magnética (MRS) registra de manera no invasiva las señales de protones en función de su frecuencia de resonancia ${ }^{28}$. La intensidad de la señal en las frecuencias correspondientes al agua o la grasa se pueden cuantificar, infiriéndose la fracción de señal de grasa ${ }^{29}$. Sin embargo, MRS tiene una aplicabilidad escasa ya que requiere métodos sofisticados para su procesamiento. Otra de las técnicas mediante RM es el Proton Density Fat Fraction (PDFF), capaz de medir la densidad de protones de hidrógeno de la grasa normalizada a partir de la densidad total de protones de hidrógeno de todas las especies de protones móviles. Las principales ventajas de PDFF sobre MRS son la simplicidad de uso en cualquier equipo de RM y la capacidad de cuantificar la esteatosis hepática en todo el hígado (a diferencia de la espectroscopia que lo realiza en pequeñas áreas) ${ }^{30,31}$.

\section{Fibrosis hepática}

El factor pronóstico más importante de la EHMG es la fibrosis, por lo que el objetivo principal será identificar a pacientes en riesgo de fibrosis avanzada. Para ello, se han desarrollado numerosos marcadores séricos no invasivos basados en parámetros clínicos $y$ analíticos $^{32}$. Los utilizados con mayor frecuencia son el NAFLD Fibrosis Score (NFS) y el FIB 4, ambos validados externamente y con un área bajo la curva alrededor de 0,8 para diagnosticar fibrosis avanzada $^{33}$. Recientemente se ha publicado un nuevo método no invasivo, denominado Hepamet Fibrosis Score, que ha demostrado aumentar la precisión diagnóstica significativamente respecto a los tests previos (área bajo la curva de 0,85$)^{34}$. Se calcula mediante 6 variables: albúmina, plaquetas, AST, HOMA, edad y sexo. Las ventajas que presenta sobre el resto de índices radica en la capacidad de disminuir los pacientes presentes en riesgo de fibrosis indeterminada y una mayor precisión diagnóstica en pacientes con edad avanzada, obesidad o DM.

Los índices séricos para la detección de la fibrosis deben ser apoyados por técnicas complementarias de imagen. Debido a su sencillez y adecuada precisión, la elastografía transitoria es la herramienta más utilizada para descartar grados de fibrosis avanzada, así como para la monitorización de la misma. Se deben realizar al menos 10 mediciones con un porcentaje de validez mayor del $60 \%$ y un IQR inferior al 30\%.

Por último, la elastografía por resonancia magnética es la técnica de imagen más precisa para evaluar el grado de fibrosis hepática ${ }^{37,38}$. El principal inconveniente de la misma es el elevado coste económico. Por este motivo, se reserva a casos en los que sea de especial importancia el establecimiento exacto del grado de fibrosis y para ensayos clínicos.

\section{Aproximación terapéutica de la EHMG}

\section{Intervención en el estilo de vida}

La intervención en el estilo de vida supone la primera línea de tratamiento en pacientes con EHMG. Dentro de dicha intervención, se incluye el abandono del sedentarismo, la actividad física moderada y la implementación de una dieta saludable ${ }^{39}$.

El sedentarismo, definido como la inactividad física durante la mayor parte de vigilia, está asociado con obesidad, DM, resistencia a la insulina, Smet y enfermedad cardiovascular ${ }^{40}$. Así, estudios experimentales muestran que períodos de inactividad física prolongada y la falta de contracción de los músculos de las extremidades inferiores induce anomalías metabólicas al suprimir la lipoproteína lipasa y la insulina del músculo esquelético, que a su vez se asocia con la resistencia insulínica periférica ${ }^{41,42}$. De esta manera, se demuestra una asociación directa entre el sedentarismo y la EHMG; hipótesis que quedó demostrada por Ryu et al., quienes confirmaron que el sedentarismo incrementaba la prevalencia de EHMG en función del tiempo de inactividad ${ }^{43}$. Además, otros estudios prospectivos han demostrado que los individuos físicamente activos son menos propensos a desarrollar complicaciones metabólicas, como la resistencia a la insulina o $\mathrm{DM}^{44}$ (factores de riesgo de EHMG), por lo que se sugiere que una actividad física mínima puede proporcionar beneficios en estos pacientes.

Por su parte, el ejercicio físico se define como un conjunto de actividades planificadas y repetitivas que se realizan con el objetivo de mejorar la forma física. En la actualidad, el ejercicio físico se considera uno de los pilares fundamentales para el tratamiento de la EHGNA, independientemente de la pérdida de peso, ya que mejora el daño hepático y las características histológicas de la enfermedad, con reducción de hasta un $20-30 \%$ de la cantidad de lípidos intrahepático ${ }^{45}$. En diversos estudios se ha comprobado que no existen diferencias entre el ejercicio físico aeróbico y el de resistencia ${ }^{46}$.En particular, en un metaanálisis publicado recientemente ${ }^{47}$, se objetivó cómo tanto el ejercicio aeróbico como el de resistencia mejoraron la esteatosis hepática sin diferencias significativas en la duración, frecuencia o período de ejercicio. Por ello, en pacientes con dificultad para el ejercicio aeróbico, se puede recomendar la realización de ejercicio físico de resistencia. En estudios a largo plazo, se ha observado cómo el ejercicio físico mantenido durante 12 meses es capaz de inducir una disminución de grasa hepática, obesidad abdominal y presión arterial hasta un año después de la intervención ${ }^{48}$.

$\mathrm{Si}$ bien es cierto que el ejercicio físico tiene un efecto significativo sobre la EHMG, sus efectos son parciales en comparación con la pérdida de peso, por lo que hay que tener en cuenta que reducir el mismo mediante dieta producirá mayor beneficio que el ejercicio físico como única intervención ${ }^{49}$. Vilar-Gómez et al. ${ }^{50}$, en un estudio prospectivo, sometió a 293 pacientes con esteatohepatitis (confirmada histológicamente) a dieta hipocalórica baja en grasas y ejercicio físico (caminar $200 \mathrm{~min} / \mathrm{semana}$ ) durante 52 semanas, con sesiones instructivas cada 8 semanas. Con una pérdida del $5 \%$ de peso, el $58 \%$ de los pacientes presentaron resolución de la esteatohepatitis y un $88 \%$ reducción de 2 puntos en el NAS score. Además, en los pacientes que perdieron más del $10 \%$ de su peso, el $100 \%$ presentaron mejoría de la esteatosis, el $90 \%$ resolución de la 


\begin{tabular}{|c|c|c|}
\hline Guias de práctica clínica & Objetivo de pérdida de peso & Restricción dietética \\
\hline EASI-EASD-EASO (1) & $\begin{array}{l}\text { Reducción del } 7-10 \% \rightarrow \\
\text { mejoria de enzimas } \\
\text { hepáticas e histologia. }\end{array}$ & $\begin{array}{l}\text { Reducción energética de 500-1,000 } \\
\mathrm{kcal} \rightarrow \text { pérdida de peso de } 500- \\
1,000 \mathrm{~g} / \text { semana. } \\
\text { Ingesta de grasas baja/moderada y } \\
\text { de carbohidratos moder ada/alta. } \\
\text { Dietas cetogenicas bajas en } \\
\text { carbohidratos / altas en proteínas. } \\
\text { Evitar las bebidas y alimentos que } \\
\text { contienen fructosa. }\end{array}$ \\
\hline AASLD (2) & $\begin{array}{l}\text { Reducción del } 3-5 \% \rightarrow \\
\text { mejoria de la esteatosis. } \\
\text { Reducción del } 7 \text { - } 10 \% \rightarrow \\
\text { mejoria histológica del } \\
\text { NASH, Induyendo fibrosis. }\end{array}$ & $\begin{array}{l}\text { Dieta mediterránea. } \\
\text { Dieta hipocalórica (reducción diaria } \\
\text { de 500-1,000 kcal). }\end{array}$ \\
\hline AEEH (3) & Reducción $\geq 7 \%$. & $\begin{array}{l}\text { Dieta mediterránea. } \\
50-60 \% \text { de hidratos de carbono y } 20 \text { - } \\
25 \% \text { de lipidos. } \\
\text { Evitar bebidas y alimentos } \\
\text { industriales que contengan fructosa. }\end{array}$ \\
\hline
\end{tabular}

Figura 1

Recomendaciones sobre pérdida de peso y restricción dietética.

esteatohepatitis y el $81 \%$ redujeron al menos un grado de fibrosis hepática.

Independientemente de que la dieta sea hipo o isocalórica, la composición de la misma es determinante para la EHMG, siendo la dieta mediterránea la considerada más efectiva ${ }^{51}$. Así, la dieta mediterránea se caracteriza por alto consumo de frutas, verduras, legumbres, frutos secos, cereales, aceite de oliva (como fuente principal de grasa), y una baja ingesta de carnes rojas, carnes procesadas y dulces. Aunque es una dieta rica en grasas, estas son principalmente grasas monoinsaturadas y poliinsaturadas con omega-3, jugando un papel beneficioso, no sólo en la EHMG, sino también en el síndrome metabólico y en la enfermedad cardiovascular $^{52}$. Se ha demostrado que la dieta mediterránea reduce la esteatosis hepática y mejora la sensibilidad a la insulina en comparación con una dieta baja en grasas y alta en carbohidratos, independientemente de la pérdida de peso ${ }^{53}$.

En conclusión, la intervención en el estilo de vida es el primer paso en el tratamiento de la EHMG, basada en una dieta mediterránea y ejercicio físico adaptado a la capacidad individual, teniendo en cuenta que la pérdida de peso resulta enormemente efectiva para la mejoría e incluso resolución de la misma. Las recomendaciones de las principales guías de práctica clínica quedan recogidas en las figuras 1 y 2.

\section{Fármacos en ensayos clínicos}

En la actualidad existen numerosos fármacos en investigación para el tratamiento de la EHMG. En esta revisión discutiremos los resultados de los fármacos que han completado al menos sus ensayos clínicos Fase II.

\section{Elafibranor}

Elafibranor es un fármaco bien tolerado que actúa como agonista dual de los receptores PPAR $\alpha$ y $\delta^{54}$. Los receptores PPAR actúan como factores de transcripción, regulando tanto el metabolismo lipídico y glucídico, como la homeostasis energética

\begin{tabular}{|l|l|}
\hline Guias de práctica clinica & Actividad fisica \\
\hline EASL-EASD-EASO (1) & $\begin{array}{l}\text { Actividad fisica aeróbica de intensidad moderada en 3-5 } \\
\text { sesiones para un total de 150-200 min / semana. } \\
\text { El entrenamiento de resistencia tambièn es efectivo. } \\
\text { La actividad fisica tiene una relación dosis-respuesta. }\end{array}$ \\
\hline AASLD (2) & $\begin{array}{l}\text { El ejercicio de intensidad moderada mejora la esteatosis } \\
\text { hepätica. }\end{array}$ \\
\hline AEEH (3) & $\begin{array}{l}\text { Actividad física (150-200 min a la semana) mejora las } \\
\text { alteraciones metabölicas y la esteatosis hepätica. }\end{array}$ \\
\hline
\end{tabular}

1. European Association for the Study of the Liver (EASL), European Association for the Study of Diabetes (EASD), European Association for the Study of Obesity (EASO). EASLEASD-EASO Clinical Practice Guidelines for the management of non-alcoholic fatty liver disease. J Hepatol. 2016 Jun;64(6):1388-402.

2. Chalasani N, Younossi Z, Lavine JE, Chariton M, Cusi K, Rinella M, et al. The diagnosis and management of nonalcoholic fatty liver disease: Practice guidance from the and management of nonalcoholic fatty liver disease: Practice guidance from the 2018;67(1):328-57. Available from: http://doi.wiley.com/10.1002/hep.29367

3. Aller R, Fernández-Rodriguez $\mathrm{C}$, lo lacono $\mathrm{O}_{\mathrm{f}}$ Bah̆ares $\mathrm{R}$, Abad J, Carrión JA, et al. Documento de consenso. Manejo de la enfermedad hepática grasa no alcohólica [EHGNA], Guia de práctica clinica. Gastroenterol Hepatol. 2018 May;41[5]:328-49.

Figura 2

Recomendaciones sobre actividad física.

y la respuesta inflamatoria ${ }^{55}$. En 2011 se empezó a evaluar como posible tratamiento para la EHGNA, comprobando que mejoraba tanto la sensibilidad a la insulina como la dislipemia, disminuyendo a su vez los valores de las enzimas hepáticas ${ }^{56}$.Posteriormente, elafibranor demostró un efecto protector en el hígado de ratas para la esteatosis, la inflamación y la fibrosis, además de mejorar los marcadores de disfunción hepática, disminuir la acumulación de lípidos intrahepáticos e inhibir expresión génica de la cascada inflamatoria y profibrótica ${ }^{57}$. Basándose en los resultados anteriores, Ratziu et al. ${ }^{58}$ realizaron un ensayo clínico fase $\mathrm{Ilb}$ en 2016 donde aleatorizaron a pacientes con esteatohepatitis confirmada histológicamente (sin cirrosis), a recibir elafibranor $80 \mathrm{mg}, 120 \mathrm{mg}$ o placebo durante un año. La dosis de $120 \mathrm{mg}$ fue superior a placebo, tanto en la resolución histológica de esteatohepatitis (sin empeorar la fibrosis) como en la mejoría de NAS score ( $20 \%$ vs. $11 \%$; $p=0,018)$. Las enzimas hepáticas, el perfil lipídico y glucídico, y los marcadores de inflamación sistémica también se redujeron significativamente. Es importante destacar que, aunque el fármaco fue bien tolerado, se observó un ligero incremento de las cifras de creatinina (reversible tras la suspensión del mismo). En la actualidad se está llevando a cabo un ensayo clínico fase III que evalúa la eficacia y seguridad de elafibranor en pacientes con esteatohepatitis no alcohólica que finalizará en 2021 (ClinicalTrials.gov: NCT02704403).

\section{Ácido obeticólico}

El receptor FXR regula tanto el metabolismo del colesterol, mediante la síntesis de ácidos biliares, como el metabolismo glucídico y lipídico, al suprimir la expresión génica de genes implicados en la gluconeogénesis y el metabolismo de las lipoproteínas ${ }^{59,60}$. El receptor FXR juega también un papel importante en la cascada inflamatoria, ya que su activación reduce la expresión de citoquinas proinflamatorias en los macrófagos ${ }^{61}$. Todo ello hace que el ácido obeticólico (OCA), agonista de dicho receptor, sea un potencial fármaco que puede aportar grandes beneficios en pacientes con EHMG. En 2015 se llevó a cabo el ensayo clínico FLINT (Fase II) que incluyó pacientes con esteatohepatitis no alcohólica (sin cirrosis) con el objetivo de evaluar el tratamiento con OCA oral ( $25 \mathrm{mg}$ al día) durante 72 semanas ${ }^{62}$. Se comprobó que el $45 \%$ de los pacientes del grupo OCA mejoraron 
su histología hepática (definida como mejoría > 2 puntos en NAS score, sin empeoramiento de la fibrosis) en comparación con el $21 \%$ de pacientes del grupo placebo $(p=0,0002)$, incluyendo además mayor beneficio en términos de esteatosis hepática, inflamación, balonización y fibrosis. Sin embargo, la proporción de pacientes con resolución de la esteatohepatitis no difirió en ambos grupos. En otro ensayo clínico fase II, se evaluó el impacto de OCA sobre la sensibilidad a la insulina en pacientes con EHMG y DM tipo 2, evidenciándose una mejoría del $24 \%$ en pacientes tratados frente a un empeoramiento del $5 \%$ en aquellos que recibieron placebo ${ }^{63}$. En cuanto a efectos adversos, el fármaco presenta prurito como evento adverso más frecuente ${ }^{64}$, así como aumento del colesterol total y LDL (0,38 mmol/L (IC95\%: 0,16-0,60) p=0,0009; 0,45 mmol/L (IC95\%: $0,26-0,65) p=0,0001$, respectivamente) y disminución del HDL $(-0.06$ $\mathrm{mmol} / \mathrm{L}$ (IC95\%: -0,10-0,01) p=0,01), con resolución de los mismos al finalizar el tratamiento.

Por todo ello, se necesitan más estudios para conocer y evaluar el efecto del fármaco en pacientes con EHMG, tanto en la resolución de la enfermedad como en los posibles efectos adversos que puedan aparecer. Así, se está llevando a cabo el estudio REGENERATE y el estudio REVERSE, ensayos clínicos en fase III, con el fin de estudiar la seguridad y la eficacia del OCA en pacientes con esteatohepatitis no alcohólica a largo plazo con fibrosis $2 / 3$ y cirrosis hepática, respectivamente (ClinicalTrials.gov: NCT02548351, NCT03439254).

\section{Liraglutide}

GLP-1 es una hormona intestinal cuya secreción depende de la presencia de alimentos ricos en carbohidratos y grasas en la luz del intestino delgado ${ }^{65}$; desempeña múltiples funciones en la homeostasis metabólica, como la secreción de insulina al promover el crecimiento, diferenciación y regeneración de la célula beta pancreática, la disminución del vaciamiento gástrico y del apetito, la disminución de la producción hepática de glucosa y la mejora de la sensibilidad periférica a la insulina, así como el aumento del gasto cardíaco ${ }^{66,67}$. Liraglutide es un análogo GLP-1 de larga duración (resistente a la enzima dipeptidil peptidasa-4) aprobado en pacientes con DM tipo 2, con gran eficacia en el control glucémico y en la pérdida de peso. Dado que la DM es un importante componente del síndrome metabólico y está asociada con el desarrollo de la EHMG, los efectos de liraglutide pueden ser de utilidad en estos pacientes. En el ensayo clínico LEAN (Fase II) ${ }^{68}$ se evaluó la eficacia de liraglutide frente a placebo durante 48 semanas en pacientes con esteatohepatitis confirmada histológicamente. Nueve (39\%) de los 23 pacientes del grupo tratado con liraglutide tuvieron resolución completa de la esteatohepatitis sin empeoramiento de la fibrosis, en comparación con 2 (9\%) de 22 pacientes del grupo placebo. Además, dos (9\%) de 23 pacientes en el grupo de liraglutide versus ocho (36\%) de 22 pacientes en el grupo de placebo tuvieron progresión de fibrosis. También se obtuvieron datos de mejoría en el peso y cifras de glucemia y lipogénesis. Aunque son necesarios más estudios para valorar los resultados a largo plazo, los hallazgos de este tipo de fármacos (agonistas GLP-1) son bastante prometedores.

\section{Cenicriviroc}

Las células de Kupffer juegan un importante papel en la patogenia de la lesión hepática, ya que son responsables de la activación de las células estrelladas a través de la producción de citoquinas pro-inflamatorias y pro-fibróticas ${ }^{69}$, promoviendo así la fibrosis. CCR2 y CCR5 son receptores de quimiocinas que se expresan principalmente en células inmunes, como las células de Kupffer, activando la cascada anteriormente descrita. Cenicriviroc actúa inhibiendo dichos receptores, demostrando en modelos animales que disminuye la fibrosis y la inflamación inducida por la dieta ${ }^{70}$. En el ensayo clínico Fase Ilb CENTAUR ${ }^{71}$ se evaluó la eficacia de cenicriviroc $150 \mathrm{mg}$ frente a placebo en 289 pacientes con esteatohepatitis (NAS score $\geq 4$ con $\geq 1$ en cada componente) y fibrosis hepática en estadío no cirrótico. Tras finalizar un año de tratamiento, la proporción de pacientes que presentaron $\geq 2$ puntos de mejora en NAS score con $\geq 1$ punto de reducción en inflamación sin empeoramiento de la fibrosis fue similar en ambos grupos ( $16 \%$ con cenicriviroc vs. $19 \%$ placebo; $p=0,52$ ). De forma similar, la resolución de esteatohepatitis sin empeoramiento de la fibrosis también fue similar en ambos grupos ( $8 \%$ vs. $6 \% ; p=0,49$ ). En cambio, la mejora en la fibrosis $\geq 1 \sin$ empeoramiento de la esteatohepatitis fue mayor en pacientes que recibieron cenicriviroc $(20 \%$ vs. $10 \% ; p=0,02)$. Los biomarcadores de inflamación sistémica (PCR, IL-6, IL-1 $\beta$ y fibrinógeno) y de activación de monocitos ( $\mathrm{SCD} 14$ ) disminuyeron en pacientes tratados con cenicriviroc. Además, la tolerabilidad y la seguridad fueron comparables a placebo. Actualmente, se está llevando a cabo un ensayo clínico en fase III para comprobar su eficacia en pacientes con esteatohepatitis y fibrosis (ClinicalTrials.gov: NCT03028740).

\section{Selonsertib}

La enzima ASK1 (activada por marcadores de inflamación como el TNF $\alpha$ ) es capaz de promover fibrosis hepática a través de la vía p38 / JNK ${ }^{72}$. Selonsertib, inhibidor selectivo de ASK1, ha demostrado en modelos animales mejorar significativamente la esteatosis hepática, la inflamación y la fibrosis asociados a esteatohepatitis $^{73}$. Loomba et al realizaron un ensayo clínico fase II en el que se evaluó la seguridad y la eficacia de selonsertib, solo o en combinación con simtuzumab, en pacientes con esteatohepatitis no alcohólica y fibrosis hepática en estadio 2 o $3^{74}$. Tras 24 semanas de tratamiento, el 43\% (13/30 pacientes) de los pacientes que recibieron $18 \mathrm{mg}$ de selonsertib y el $30 \%$ (8/27 pacientes) de los que recibieron $6 \mathrm{mg}$ presentaron reducción de al menos un estadío de fibrosis hepática, frente al 20\% (2/10 pacientes) de los que recibieron únicamente simtuzumab. Ante estos resultados, en 2017 se iniciaron dos ensayos clínicos Fase III, STELLAR-3 y STELLAR-4, donde se evaluaba la eficacia de selonsertib en pacientes con fibrosis avanzada y cirrosis, respectivamente ${ }^{75,76}$. Sin embargo, ambos ensayos se suspendieron en 2019 debido a que en el análisis intermedio no se consiguió el objetivo primario. Así, en el STELLAR-4 se objetivó que el $14,4 \%$ de los pacientes tratados con selonsertib a $18 \mathrm{mg}$ y el $12,5 \%$ tratados con la dosis de $6 \mathrm{mg}$ lograron regresión de la cirrosis hepática, en comparación con $12,8 \%$ de los que recibieron placebo. De igual manera, en el STELLAR-3, el 9,3\% de los pacientes tratados con selonsertib $18 \mathrm{mg}$ y el $12,1 \%$ de los pacientes tratados con $6 \mathrm{mg}$ lograron una mejoría de al menos un estadío de fibrosis hepática tras 48 semanas de tratamiento, frente a $13,2 \%$ con placebo.

\section{Conclusiones}

La enfermedad hepática metabólica grasa es una entidad cada vez más frecuente, siendo indispensable distinguir aquellos sujetos que van a evolucionar y progresar (peor pronóstico) de 
los que permanecerán estables. Para ello, se han desarrollado una serie de métodos no invasivos que permiten estadificar a los pacientes en bajo o alto riesgo de fibrosis hepática avanzada, según una combinación de parámetros analíticos, epidemiológicos, de imagen y elastográficos. El pilar fundamental del tratamiento es la intervención en el estilo de vida, modificando el patrón de la dieta y la ingesta calórica, así como implementando medidas encaminadas hacia la realización de ejercicio físico de forma regular. En un segundo escalón quedan las intervenciones farmacológicas, ya que tienen que finalizar los ensayos clínicos Fase III en marcha que permitirán elucidar quiénes se beneficiarán finalmente de estos tratamientos.

\section{Bibliografía}

1. Younossi ZM, Koenig AB, Abdelatif D, Fazel Y, Henry L, Wymer M. Global epidemiology of nonalcoholic fatty liver disease- Metaanalytic assessment of prevalence, incidence, and outcomes. Hepatology 2016;64:73-84

2. Lazo M, Clark JM. The epidemiology of nonalcoholic fatty liver disease: A global perspective. Semin Liv Dis. 2008;28:380-385.

3. EASL, EASD, EASO. EASL-EASD-EASO Clinical Practice Guidelines for the management of non-alcoholic fatty liver disease. J Hepatol. 2016;64(6):13881402.

4. Sasaki A, Nitta H, Otsuka K, Umemura A, Baba S, Obuchi T, et al. Bariatric surgery and non-alcoholic fatty liver disease: current and potential future treatments. Front Endocrinol 2014;5:164.

5. Bril F, Cusi K. Management of nonalcoholic fatty liver disease in patients with type 2 diabetes: a call to action. Diabetes Care 2017;40:419-430.

6. Doycheva I, Cui J, Nguyen P, Costa EA, Hooker J, Hofflich H, et al. Non-invasive screening of diabetics in primary care for NAFLD and advanced fibrosis by MRI and MRE. Aliment Pharmacol Ther 2016;43:83-95.

7. Wu KT, Kuo PL, Su SB, Chen YY, Yeh ML, Huang Cl, et al. Non-alcoholic fatty liver disease severity is associated with the ratios of total cholesterol and triglycerides to high-density lipoprotein cholesterol. J Clin Lipidol 2016;10:420425 .

8. Bargiggia S, Maconi G, Elli M, Molteni P, Ardizzone S, Parente F et al. Sonographic prevalence of liver steatosis and biliary tract stones in patients with inflammatory bowel disease: study of 511 subjects at a single center. J Clin Gastroenterol 2003;36(5):417-420.

9. Glassner K, Malaty HM, Abraham BP. Epidemiology and Risk Factors of Nonalcoholic Fatty Liver Disease Among Patients with Inflammatory Bowel Disease. Inflamm Bowel Dis 2017;23(6):998-1003.

10. Sourianarayanane A, Garg G, Smith TH, Butt MI, McCullough AJ, Shen B. Risk factors of non-alcoholic fatty liver disease in patients with inflammatory bowel disease. J Crohns Colitis 2013;7(8):279-285.

11. Zou ZY, Shen B, Fan JG. Systematic Review with Meta-analysis: Epidemiology of Nonalcoholic Fatty Liver Disease in Patients with Inflammatory Bowel Disease. Inflamm Bowel Dis 2019;25(11):1764-1772.
12. Sartini A, Gitto S, Bianchini M, Verga MC, Di Girolamo M, Bertani A et al. Non-alcoholic fatty liver disease phenotypes in patients with inflammatory bowel disease. Cell Death Dis 2018;9(2):87.

13. Carrascosa JM, Bonanad C, Dauden E, Botella R, Olveira-Martin A. Psoriasis and Nonalcoholic Fatty Liver Disease. Actas Dermosifiliogr 2017;108(6):506514.

14. Candia R, Ruiz A, Torres-Robles R, Chávez-Tapia N, Méndez-Sánchez N, Arrese M. Risk of non-alcoholic fatty liver disease in patients with psoriasis: a systematic review and meta-analysis. J Eur Acad Dermatol Venereol 2015;29(4):656-662.

15. Wu J, Yao XY, Shi RX, Liu SF, Wang XY. A potential link between polycysticovary syndrome and non-alcoholic fatty liver disease: An update meta-analysis. Reprod. Health 2018;15(1):77.

16. Durán-Vian C, Arias-Loste MT, Hernández JL, Fernández V, González M, Iruzubieta $\mathrm{P}$ et al. High prevalence of non-alcoholic fatty liver disease among hidradenitis suppurativa patients independent of classic metabolic risk factors. J Eur Acad Dermatol Venereol 2019;33(11):2131-2136.

17. Bedossa P. Intraobserver and interobserver variations in liver biopsy interpretation in patients with chronic hepatitisC. Hepatology. 1994;20:15-20.

18. Vuppalanchi R, Unalp A, Van Natta ML, Cummings OW, Sandrasegaran KE, Hameed T, et al. Effects of liver biopsy sample length and number of readings on sampling variability in nonalcoholic fatty liver disease. Clin Gastroenterol Hepatol 2009;7:481-486.

19. Aller R, Fernández-Rodríguez C, Lo lacono O, Bañares R, Abad J, Carrión JA et al. Consensus document. Management of non-alcoholic fatty liver disease (NAFLD). Clinical practice guideline. Gastroenterol Hepatol 2018;41(5):328349.

20. Harman DJ, Ryder SD, James MW, Wilkes EA, Card TR, Aithal GP et al. Obesity and type 2 diabetes are important risk factors underlying previously undiagnosed cirrhosis in general practice: a cross-sectional study using transient elastography. Aliment Pharmacol Ther 2018;47:504-515.

21. Mofrad P, Contos MJ, Haque M, Sargeant C, Fisher RA, Luketic VA, et al. Clinical and histologic spectrum of nonalcoholic fatty liver disease associated with normal ALT values. Hepatology 2003;37(6):1286-1292.

22. Stern C, Castera L. Non-invasive diagnosis of hepatic steatosis. Hepatol Int 2017;11(1):70-78.

23. Fedchuk L, Nascimbeni F, Pais R, Charlotte F, Housset C, Ratziu V et al. Performance and limitations of steatosis biomarkers in patients with nonalcoholic fatty liver disease. Aliment Pharmacol Ther 2014;40:1209-1222.

24. Lee SS, Park SH, Kim HJ, Kim SY, Kim MY, Kim DY, et al. Non-invasive assessment of hepatic steatosis: prospective comparison of the accuracy of imaging examinations. J Hepatol 2010;52:579-585.

25. Baršić N, Lerotić I, Smirčić-Duvnjak L, Tomašić V, Duvnjak M. Overview and developments in noninvasive diagnosis of nonalcoholic fatty liver disease. World J Gastroenterol 2012;18:3945-3954. 
26. Mottin CC, Moretto M, Padoin AV, Swarowsky AM, Toneto MG, Glock L, et al. The role of ultrasound in the diagnosis of hepatic steatosis in morbidly obese patients. Obes Surg 2004;14:635-637.

27. Karlas T, Petroff D, Sasso M, Fan JG, Mi YQ, de Lédinghen V, et al. Individual Patient Data Meta-Analysis of Controlled Attenuation Parameter (CAP) Technology for Assessing Steatosis. J Hepatol 2017;66(5):1022-1030.

28. Reeder SB, Cruite I, Hamilton G, Sirlin CB. Quantitative assessment of liver fat with magnetic resonance imaging and spectroscopy. J Magn Reson Imaging 2011;34:729-49.

29. Loomba R. Role of imaging-based biomarkers in NAFLD: Recent advances in clinical application and future research directions. J Hepatol 2018;68(2):296304.

30. Meisamy S, Hines CD, Hamilton G, Sirlin CB, McKenzie CA, Yu H, et al. Quantification of hepatic steatosis with T1-independent, T2-corrected MR imaging with spectral modeling of fat: blinded comparison with MR spectroscopy. Radiology 2011;258(3):767-775.

31. Di Martino M, Pacifico L, Bezzi M, Di Miscio R, Sacconi B, Chiesa C, et al. Comparison of magnetic resonance spectroscopy, proton density fat fraction and histological analysis in the quantification of liver steatosis in children and adolescents. World J Gastroenterol 2016;22(39):8812-8819.

32. Boursier J, Vergniol J, Guillet A, Hiriart JB, Lannes A, Le Bail B, et al. Diagnostic accuracy and prognostic significance of blood fibrosis tests and liver stiffness measurement by FibroScan in non-alcoholic fatty liver disease. J Hepatol 2016;65(3):570-578.

33. Castera L. Diagnosis of non-alcoholic fatty liver disease/ non-alcoholic steatohepatitis: Non-invasive tests are enough. Liver Int 2018;38:67-70.

34. Ampuero J, Pais R, Aller R, Gallego-Durán R,Crespo J,García-Monzón C, et al. Development and Validation of Hepamet Fibrosis Scoring System A Simple, Noninvasive Test to Identify Patients With Nonalcoholic Fatty Liver Disease With Advanced Fibrosis. Clin Gastroenterol Hepatol (2019), doi:10.1016/j. cgh.2019.05.051.

35. Karlas T, Petroff D, Sasso M, Fan JG, Mi YQ, de Lédinghen V, et al. Impact of controlled attenuation parameter on detecting fibrosis using liver stiffness measurement. Aliment Pharmacol Ther 2018;47(7):989-1000.

36. Puigvehí $\mathrm{M}$, Broquetas $\mathrm{T}$, Coll $\mathrm{S}$, Garcia-Retortillo $\mathrm{M}$, Cañete $\mathrm{N}$, Fernández $\mathrm{R}$, et al. Impact of anthropometric features on the applicability and accuracy of FibroScan ${ }^{\circledast}(\mathrm{M}$ and $\mathrm{XL}$ ) in overweight/obese patients. J. Gastroenterol. Hepatol. 2017;32:1746-1753.

37. Hsu C, Caussy C, Imajo K, Chen J, Singh S, Kaulback K, et al. Magnetic Resonance vs Transient Elastography Analysis of Patients with Nonalcoholic Fatty Liver Disease: A Systematic Review and Pooled Analysis of Individual Participants. Clin Gastroenterol Hepatol 2019;17(4):630-637.

38. Friedrich-Rust M, Ong MF, Martens S, Sarrazin C, Bojunga J, Zeuzem S, et al. Performance of transient elastography for the staging of liver fibrosis: a metaanalysis. Gastroenterology 2008;134(4):960-974.

39. Romero-Gómez M, Zelber-Sagi S, Trenell M. Treatment of NAFLD with diet, physical activity and exercise. J Hepatol. 2017 Oct;67(4):829-46.
40. Biswas A, Oh PI, Faulkner GE, Bajaj RR, Silver MA, Mitchell MS, et al. Sedentary time and its association with risk for disease incidence, mortality, and hospitalization in adults: a systematic review and meta-analysis. Ann Intern Med. 2015 Jan 20;162(2):123-32.

41. Hamilton MT, Hamilton DG, Zderic TW. Role of low energy expenditure and sitting in obesity, metabolic syndrome, type 2 diabetes, and cardiovascular disease. Diabetes. 2007 Nov 1;56(11):2655-67.

42. Wang $H$, Knaub LA, Jensen DR, Young Jung D, Hong E-G, Ko H-J, et al. Skeletal muscle-specific deletion of lipoprotein lipase enhances insulin signaling in skeletal muscle but causes insulin resistance in liver and other tissues. Diabetes. 2009 Jan 1;58(1):116-24.

43. Ryu S, Chang Y, Jung H-S, Yun KE, Kwon M-J, Choi Y, et al. Relationship of sitting time and physical activity with non-alcoholic fatty liver disease. J Hepatol. 2015 Nov;63(5):1229-37.

44. Snowling NJ, Hopkins WG. Effects of Different Modes of Exercise Training on Glucose Control and Risk Factors for Complications in Type 2 Diabetic Patients: A meta-analysis. Diabetes Care. 2006 Nov 1;29(11):2518-27.

45. Kwak M-S, Kim D. Non-alcoholic fatty liver disease and lifestyle modifications, focusing on physical activity. Korean J Intern Med. 2018 Jan 1;33(1):64-74.

46. Shamsoddini A, Sobhani V, Ghamar Chehreh ME, Alavian SM, Zaree A. Effect of Aerobic and Resistance Exercise Training on Liver Enzymes and Hepatic Fat in Iranian Men With Nonalcoholic Fatty Liver Disease. Hepat Mon. 2015 Oct 10;15(10):e31434.

47. Hashida R, Kawaguchi T, Bekki M, Omoto M, Matsuse H, Nago T, et al. Aerobic vs. resistance exercise in non-alcoholic fatty liver disease: A systematic review. J Hepatol. 2017 Jan;66(1):142-52.

48. Zhang H-J, Pan L-L, Ma Z-M, Chen Z, Huang Z-F, Sun Q, et al. Long-term effect of exercise on improving fatty liver and cardiovascular risk factors in obese adults: A 1-year follow-up study. Diabetes Obes Metab. 2017 Feb;19(2):284-9.

49. Thoma C, Day CP, Trenell MI. Lifestyle interventions for the treatment of non-alcoholic fatty liver disease in adults: A systematic review. J Hepatol. 2012 Jan;56(1):255-66.

50. Vilar-Gomez E, Martinez-Perez Y, Calzadilla-Bertot L, Torres-Gonzalez A, Gra-Oramas B, Gonzalez-Fabian L, et al. Weight Loss Through Lifestyle Modification Significantly Reduces Features of Nonalcoholic Steatohepatitis. Gastroenterology. 2015 Aug;149(2):367-378.e5.

51. Eslamparast T, Tandon P, Raman M. Dietary Composition Independent of Weight Loss in the Management of Non-Alcoholic Fatty Liver Disease. Nutrients. 2017 Jul 26;9(8):800.

52. Estruch R, Ros E, Salas-Salvadó J, Covas M-I, Corella D, Arós F, et al. Primary prevention of cardiovascular disease with a Mediterranean diet. N Engl J Med. 2013 Apr 4;368(14):1279-90.

53. Ryan MC, Itsiopoulos C, Thodis T, Ward G, Trost N, Hofferberth S, et al. The Mediterranean diet improves hepatic steatosis and insulin sensitivity in individuals with non-alcoholic fatty liver disease. J Hepatol. 2013 Jul;59(1):13843. 
54. Cariou B, Hanf R, Lambert-Porcheron S, Zair Y, Sauvinet V, Noel B, et al. Dual peroxisome proliferator- activated receptor $\alpha / \delta$ agonist GFT505 improves hepatic and peripheral insulin sensitivity in abdominally obese subjects. Diabetes Care. 2013;36(10):2923-30.

55. Quintero $P$, Arrese M. Nuclear control of inflammation and fibrosis in nonalcoholic steatohepatitis: Therapeutic potential of dual peroxisome proliferator-activated receptor alpha/delta agonism. Hepatology. 2013;58(6):1881-4.

56. Cariou B, Zaïr Y, Staels B, Bruckert E. Effects of the new dual PPAR $\alpha / \delta$ agonist GFT505 on lipid and glucose homeostasis in abdominally obese patients with combined dyslipidemia or impaired glucose metabolism. Diabetes Care. 2011;34(9):2008-14

57. Staels B, Rubenstrunk A, Noel B, Rigou G, Delataille P, Millatt LJ, et al. Hepatoprotective effects of the dual peroxisome proliferator-activated receptor alpha/delta agonist, GFT505, in rodent models of nonalcoholic fatty liver disease/nonalcoholic steatohepatitis. Hepatology. 2013;58(6):1941-52.

58. Ratziu V, Harrison SA, Francque S, Bedossa P, Lehert P, Serfaty L, et al. Elafibranor, an Agonist of the Peroxisome Proliferator-Activated Receptor- $\alpha$ and $-\delta$, Induces Resolution of Nonalcoholic Steatohepatitis Without Fibrosis Worsening. Gastroenterology. 2016;150(5):1147-1159e5.

59. Lambert G. The Farnesoid X-receptor Is an Essential Regulator of Cholesterol Homeostasis. J Biol Chem. 2003 Jan 17 [cited 2017 Feb 2];278(4):2563-70.

60. Ma K, Saha PK, Chan L, Moore DD. Farnesoid X receptor is essential for normal glucose homeostasis. J Clin Invest. 2006;116(4):1102.

61. Wang Y-D, Chen W-D, Wang M, Yu D, Forman BM, Huang W. Farnesoid $X$ receptor antagonizes nuclear factor $\mathrm{KB}$ in hepatic inflammatory response. Hepatology. 2008 Nov [cited 2017 Feb 18];48(5):1632-43.

62. Neuschwander-Tetri BA, Loomba R, Sanyal AJ, Lavine JE, Van Natta $\mathrm{ML}$, Abdelmalek MF, et al. Farnesoid $\mathrm{X}$ nuclear receptor ligand obeticholic acid for non-cirrhotic, non-alcoholic steatohepatitis (FLINT): a multicentre, randomised, placebo-controlled trial. Lancet. 2015 Mar;385(9972):956-65.

63. Mudaliar S, Henry RR, Sanyal AJ, Morrow L, Marschall HU, Kipnes M, et al. Efficacy and safety of the farnesoid $\mathrm{x}$ receptor agonist Obeticholic acid in patients with type 2 diabetes and nonalcoholic fatty liver disease. Gastroenterology. 2013;145(3):574-582.e1.

64. Nevens F, Andreone P, Mazzella G, Strasser SI, Bowlus C, Invernizzi P, et al. A Placebo-Controlled Trial of Obeticholic Acid in Primary Biliary Cholangitis. N Engl J Med. 2016 Aug 18 [cited 2017 Feb 18];375(7):631-43.
65. Yusta B, Baggio LL, Koehler J, Holland D, Cao X, Pinnell LJ, et al. GLP1R Agonists Modulate Enteric Immune Responses Through the Intestinal Intraepithelial Lymphocyte GLP-1R. Diabetes. 2015 Jul;64(7):2537-49.

66. Escalada FJ. Fisiología del GLP-1 y su papel en la fisiopatología de la diabetes mellitus tipo 2. Med Clin (Barc). 2014 Sep;143:2-7.

67. Baggio LL, Drucker DJ. Biology of incretins: GLP-1 and GIP. Gastroenterology. 2007 May;132(6):2131-57

68. Armstrong MJ, Gaunt P, Aithal GP, Barton D, Hull D, Parker R, et al. Liraglutide safety and efficacy in patients with non-alcoholic steatohepatitis (LEAN): a multicentre, double-blind, randomised, placebo-controlled phase 2 study. Lancet (London, England). 2016 Feb;387(10019):679-90.

69. Ju C, Tacke F. Hepatic macrophages in homeostasis and liver diseases: from pathogenesis to novel therapeutic strategies. Cell Mol Immunol. 2016 May 24;13(3):316-27.

70. Lefebvre E, Moyle G, Reshef R, Richman LP, Thompson M, Hong F, et al. Antifibrotic Effects of the Dual CCR2/CCR5 Antagonist Cenicriviroc in Anima Models of Liver and Kidney Fibrosis. Sookoian SC, editor. PLoS One. 2016 Jun 27;11(6):e0158156.

71. Friedman SL, Ratziu V, Harrison SA, Abdelmalek MF, Aithal GP, Caballeria $J$, et al. A randomized, placebo-controlled trial of cenicriviroc for treatment of nonalcoholic steatohepatitis with fibrosis. Hepatology. 2018 May;67(5):175467

72. Brenner C, Galluzzi L, Kepp O, Kroemer G. Decoding cell death signals in liver inflammation. J Hepatol. 2013 Sep;59(3):583-94.

73. Di Prospero NA, Artis E, Andrade-Gordon P, Johnson DL, Vaccaro N, Xi L, et al. CCR2 antagonism in patients with type 2 diabetes mellitus: a randomized, placebo-controlled study. Diabetes Obes Metab. 2014 Nov;16(11):1055-64.

74. Loomba R, Lawitz E, Mantry PS, Jayakumar S, Caldwell SH, Arnold H, et al. The ASK1 inhibitor selonsertib in patients with nonalcoholic steatohepatitis: A randomized, phase 2 trial. Hepatology. 2018 Feb 26;67(2):549-59.

75. Gilead Announces Topline Data From Phase 3 STELLAR-3 Study of Selonsertib in Bridging Fibrosis (F3) Due to Nonalcoholic Steatohepatitis (NASH) [Internet]. [cited 2019 Dec 9]. Available from: http://natap.org/2019/ HCV/050819_01.htm

76. Gilead Announces Topline Data From Phase 3 STELLAR-4 Study of Selonsertib in Compensated Cirrhosis (F4) Due to Nonalcoholic Steatohepatitis (NASH) [Internet]. [cited 2019 Dec 9]. Available from: http://www.natap. org/2019/HCV/022719_01.htm 